\title{
Anestesia cardiovascular en cirugía no cardiaca
}

\section{Cardiovascular anesthesia in noncardiac surgery}

\author{
Evelyn Borchert'1 Katia González', Guillermo Lema
}

\begin{abstract}
Clinical evaluation remains one of the main issues while considering anesthetic and surgical risk. Different scores for cardiac evaluation in non-cardiac surgery are traditionally based on the exclusion of active cardiac conditions, the risk of surgery, the functional capacity of the patient and the presence of specific cardiac risk factors. In recent decades, new guidelines incorporate an association between cardiac biomarkers and adverse cardiac events. For the management of coronary patients receiving double antiplatelet therapy, derived for non-cardiac surgery, the risk of stent thrombosis, the consequences of delaying the surgical procedure and the risk of bleeding must be considered. At this moment, there is no evidence regarding which is the best anesthetic management that decreased peri-operative cardiovascular complications in this group of patients. This article refers to the differences in preoperative assessment for non-cardiac surgery incorporated in the guidelines of the American College of Cardiology, the American Heart Association, the European Society of Cardiology and the Canadian Cardiovascular Society. Consideration are also given to the management of coronary patients on double antiplatelet therapy and its main complications as well as intraoperative management maneuvers that may decrease cardiovascular complications.
\end{abstract}

\section{RESUMEN}

La valoración clínica sigue siendo uno de los pilares fundamentales en la evaluación del riesgo anestésico-quirúrgico. Los scores de riesgo para la evaluación cardiovascular y cirugía no cardíaca se basan tradicionalmente en la exclusión de condiciones cardíacas activas, la determinación del riesgo de cirugía, la capacidad funcional del paciente y la presencia de factores de riesgo cardíaco. En

\section{Key words:}

Preoperative risk stratification, cardiac biomarkers, double anticoagulant treatment, non-cardiac surgery

\section{Palabras clave:}

Estratificación de riesgo preoperatorio, biomarcadores cardiacos, tratamiento anticoagulante doble, cirugía no cardiaca

Departamento de Anestesiología, Pontificia Universidad Católica de Chile.

Fecha de recepción: 27 de abril de 2020

Fecha de aceptación: 16 de mayo de 2020

\section{ORCID}

https://orcid.org/0000-0001-8722-985X

Correspondencia:

Evelyn Borchert

evelynborchert@gmail.com 
las últimas décadas, nuevas guías incorporan una asociación entre los biomarcadores cardiacos y los eventos cardiacos adversos. Para el manejo de pacientes coronarios en tratamiento antiagregante doble, derivados a cirugía no cardiaca, hay que considerar el riesgo de trombosis del stent, las consecuencias de retrasar el procedimiento quirúrgico y el aumento del riesgo de hemorragia. Hasta la fecha no existe evidencia acerca de cuál es el mejor manejo anestésico que disminuya las complicaciones cardiovasculares perioperatorias en este grupo de pacientes. Este artículo, hace referencia a las diferencias de la valoración preoperatoria para cirugía no cardiaca incorporados en las guías del American College of Cardiology, la American Heart Association, la European Society of Cardiology y la Canadian Cardiovascular Society. Algunas consideraciones acerca del manejo de pacientes coronarios, terapia antiplaquetaria dual y eventuales complicaciones. Se incluyen algunas estrategias farmacológicas, así como consideraciones específicas para el perioperatorio, con el fin de reducir morbilidad de origen cardiovascular.

os cardiólogos, anestesistas y profesionales que trabajan en este tipo de procedimientos, están familiarizados, con la valoración clínica y scores de riesgo recomendadas por la American College of Cardiology (ACC), American Heart Association (AHA), la European Society of Cardiology (ESC) y la Canadian Cardiovascular Society (CSC). Todas ellas vigentes actualmente. Sin embargo, existen temas que aún generan controversia y que para resolverlos debemos adaptarlos a la práctica clínica del centro y del equipo de salud en el que nos encontramos[1]-[ 4].

Para la $A H A$ y la ECS, hay tres aspectos fundamentales para una correcta evaluación: definir factores de riesgo mediante índices y/o scores, tipo de cirugía y la capacidad funcional (Tabla 1).

Los índices clínicos de riesgo, además de evaluar el riesgo cardiaco, permiten determinar el beneficio de mayores evaluaciones, necesidad de monitorización invasiva en el intra y postoperatorio, destino para la recuperación postoperatoria, e información lo más exacta posible para pacientes y familiares.

Dos de ellos son los más aceptados (recomendación Ib): el índice de riesgo cardiaco corregido (RCRI) y el modelo de predicción del American College of Surgeons (American College of Surgeons National Surgical Quality Improvement Programa/Myocardial Infarction and Cardiac Arrest) NSQIPIMICA[1]-[3].

El $R C R[[2]] 3$,$] , fue diseñado para predecir el riesgo$ postoperatorio de infarto agudo de miocardio (IAM), edema pulmonar, fibrilación ventricular, paro cardiaco (PCR) y bloqueo aurículo-ventricular completo (BAVC). Analiza seis factores clínicos de riesgo independiente: cirugías de alto riesgo, cardiopatía isqué- mica, insuficiencia cardiaca congestiva, enfermedad cerebrovascular, diabetes mellitus insulino requirente y disfunción renal. A mayor número de factores de riesgo, mayor es la frecuencia de complicaciones cardiacas mayores (Tabla 2).

En una revisión sistemática de 24 estudios[2],[3], se observó que el índice de Lee discriminaba moderadamente bien a los pacientes de bajo riesgo, de aquellos de riesgo elevado de eventos cardiacos, tras diferentes tipos de cirugía no cardiaca. Sin embargo, no predijo eventos cardiacos ni mortalidad tras la cirugía vascular. Un aspecto discutible es que fue descrito solo para cirugías electivas y no incluyó pacientes mayores de 70 años[2],[3]. Hasta donde la literatura señala, no hay una clasificación, semejante a la descrita para cirugías de urgencias y/o emergencias.

El modelo NSQIPIMICA reporta los datos pre, intra y postoperatorios de más de 200 hospitales con respecto a las complicaciones cardiacas[1],[3]. Analizaron los factores de riesgo que se asociaban a IAM y PCR durante y hasta los 30 días posteriores a la cirugía, desarrollando y validando un score de riesgo que permitiera predecirlos. Se identificaron cinco variables predictivas: el tipo de cirugía, la clase funcional preoperatoria, creatinina plasmática mayor a 1,5 $\mathrm{mg} / \mathrm{dl}$, edad y clasificación de acuerdo a la American Society of Anesthesiology (ASA). De esta forma se desarrolló una hoja de cálculo interactiva donde se aportan los datos del paciente y se obtiene un porcentaje estimado de sufrir IAM o PCR (http://www.surgicalriskcalculator.com/miocardiaca-rrest)[1],[3]. Este modelo no considera algunas complicaciones clínicas cardiacas perioperatorias como edema pulmonar y BAVC, y a pesar de ello, tiene un poder de discrimi- 


\begin{tabular}{|c|c|c|}
\hline $\begin{array}{l}\text { *Cirugía de bajo riesgo } \\
(\text { menor a } 1 \%)\end{array}$ & $\begin{array}{l}\text { *Cirugía de riesgo intermedio } \\
\text { (entre } 1-5 \%)\end{array}$ & $\begin{array}{l}\text { *Cirugías de alto riesgo } \\
\text { (mayor a } 5 \%)\end{array}$ \\
\hline Cirugía superficial & $\begin{array}{l}\text { Intraperitoneal: esplenectomía, repara- } \\
\text { ción de hernia de hiato, colecistectomía }\end{array}$ & Cirugía aórtica y vascular mayor \\
\hline Mamas & $\begin{array}{l}\text { Carotidea sintomática (endarterecto- } \\
\text { mía o stent carotídeo) }\end{array}$ & $\begin{array}{l}\text { Revascularización abierta de extremi- } \\
\text { dades inferiores, amputación, trombo } \\
\text { embolectomía }\end{array}$ \\
\hline Dental & Angioplastía arterial periférica & Cirugía de duodeno y páncreas \\
\hline Tiroides & Reparación endovascular de aneurisma & $\begin{array}{l}\text { Resección de hígado cirugía de vías bi- } \\
\text { liares }\end{array}$ \\
\hline Ocular & Cirugía de cabeza y cuello & Esofagectomía \\
\hline Reconstructiva & $\begin{array}{l}\text { Neurológica u ortopédica mayor (ciru- } \\
\text { gía de cadera y columna) }\end{array}$ & Reparación de perforación intestinal \\
\hline $\begin{array}{l}\text { Carotidea asintomática endarterecto- } \\
\text { mía o stent carotídeo }\end{array}$ & Urológica o ginecológica mayor & Resección de glándulas suprarrenales \\
\hline Ginecológica menor & Trasplante renal & Cistectomía total \\
\hline Ortopédica menor & Intratorácica no mayor & Neumonectomía \\
\hline $\begin{array}{l}\text { Urológica menor (resección transurete- } \\
\text { ral de próstata) }\end{array}$ & & Trasplante pulmonar o hepático \\
\hline
\end{tabular}

*Se refiere al riesgo estimado (\%) de presentar un evento cardiaco (muerte y/o infarto de miocardio (IAM) a los 30 días del procedimiento).

\begin{tabular}{|c|c|c|}
\hline Número de factores de riesgo & $\begin{array}{l}\text { Frecuencia de complicaciones car- } \\
\text { diacas mayores (riesgo estimado) }\end{array}$ & $95 \%$ IC para el riesgo estimado \\
\hline 0 & $3,9 \%$ & $2,8 \%-5,4 \%$ \\
\hline 1 & $6,0 \%$ & $4,9 \%-7,4 \%$ \\
\hline 2 & $10,1 \%$ & $8,1 \%-12,6 \%$ \\
\hline$>3$ & $15 \%$ & $11,1 \%-20 \%$ \\
\hline
\end{tabular}

IC: Intervalo de confianza; *Sobre la base de estudios de validación externa de alta calidad

nación superior de predecir eventos cardiacos. Puede estar subestimado ya que al no incluir mediciones sistemáticas de niveles de troponina perioperatorios, un porcentaje no menor de los IAM pueden no haber sido detectados[4].

La evaluación de la capacidad funcional forma parte de la evaluación clínica inicial y aporta importante información sobre la posibilidad de complicaciones cardiacas y sobre la necesidad de profundizar en estudios diagnósticos. Una mala capacidad funcional, es decir, menos de 4 METs (unidad de medida del índice metabólico) cuando se asocia a cirugía torácica, presenta complicaciones cardiacas elevadas porque refleja el estado de la función cardiaca y pulmonar integradas[3]. En otras cirugías, la asociación es débil, adquiriendo especial importancia la buena clase funcional como predictor negativo. Otros autores encuentran una relación inversa entre complicaciones cardiacas mayores y número de cuadras que el paciente puede caminar. La base de datos de NSQIP mostró que los pacientes definidos como un estado funcional "dependiente" (menor a 4METs) se asociaban a un incremento significativo en el riesgo perioperatorio de muerte y/o IAM[1],[3] (Figura 1).

Con esta información se presenta a continuación los algoritmos de ambas sociedades (ESC y ACC/AHA) 


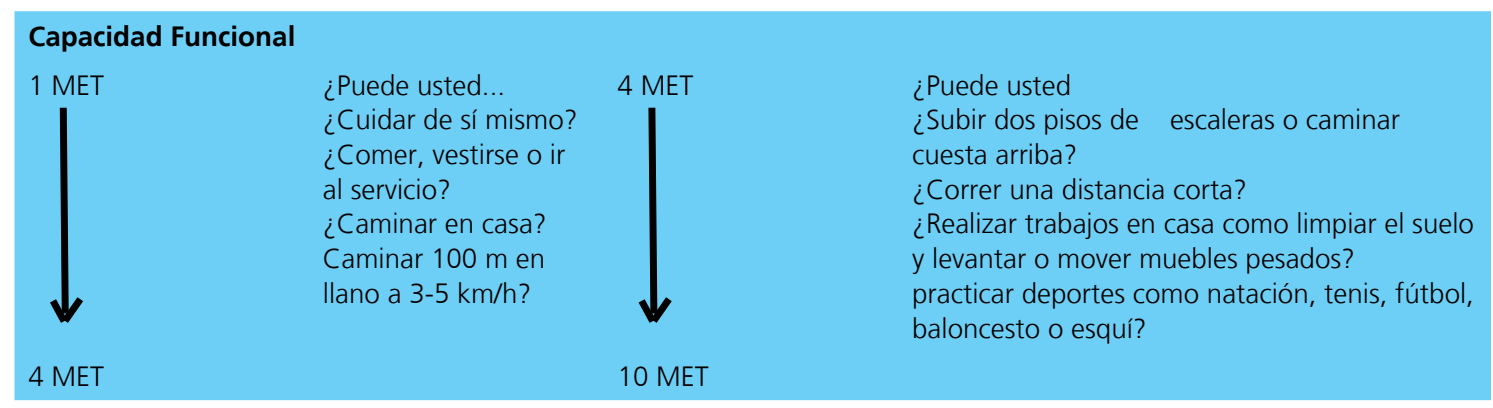

Figura 1. Estimación de la energía necesaria para varias actividades. Basada en Hlatky y cols y Fletcher y cols.

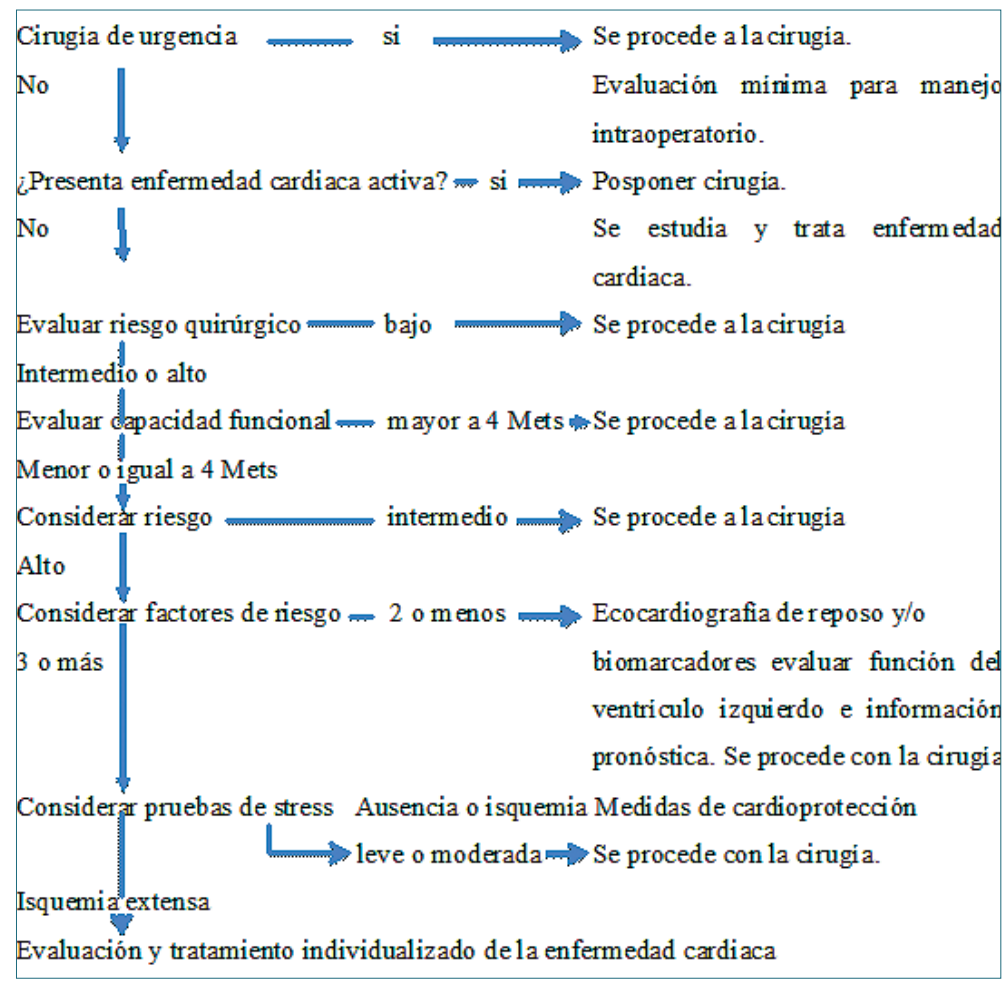

Figura 2. Algoritmo ESC.

para la práctica clínica con sus principales diferencias. Figuras 2 y 3

\section{Paso 1}

Es común a ambas y establecen que, si la cirugía es urgente, no es posible realizar tratamientos o evaluaciones cardiacas adicionales. Se procede a la cirugía y los elementos de valoración están dirigidos a optimizar el tratamiento intra y postoperatorio.

\section{Paso 2}

Ambas establecen que si hay una enfermedad car- diaca activa: síndromes coronario inestables, insuficiencia cardiaca descompensada, arritmias graves y valvulopatías sintomáticas, la cirugía debe aplazarse y el paciente debe estabilizarse. El anestesiólogo debe participar activamente ya que la decisión final puede comprometer el procedimiento quirúrgico/anestésico.

\section{Paso 3}

Se evalúa el riesgo de desencadenar un evento cardiaco. Si el riesgo es bajo (ESC), (menor de 1\%), se procede con la cirugía. Para la ACCIAHA se debe estimar el riesgo de un evento cardiaco en base a la 


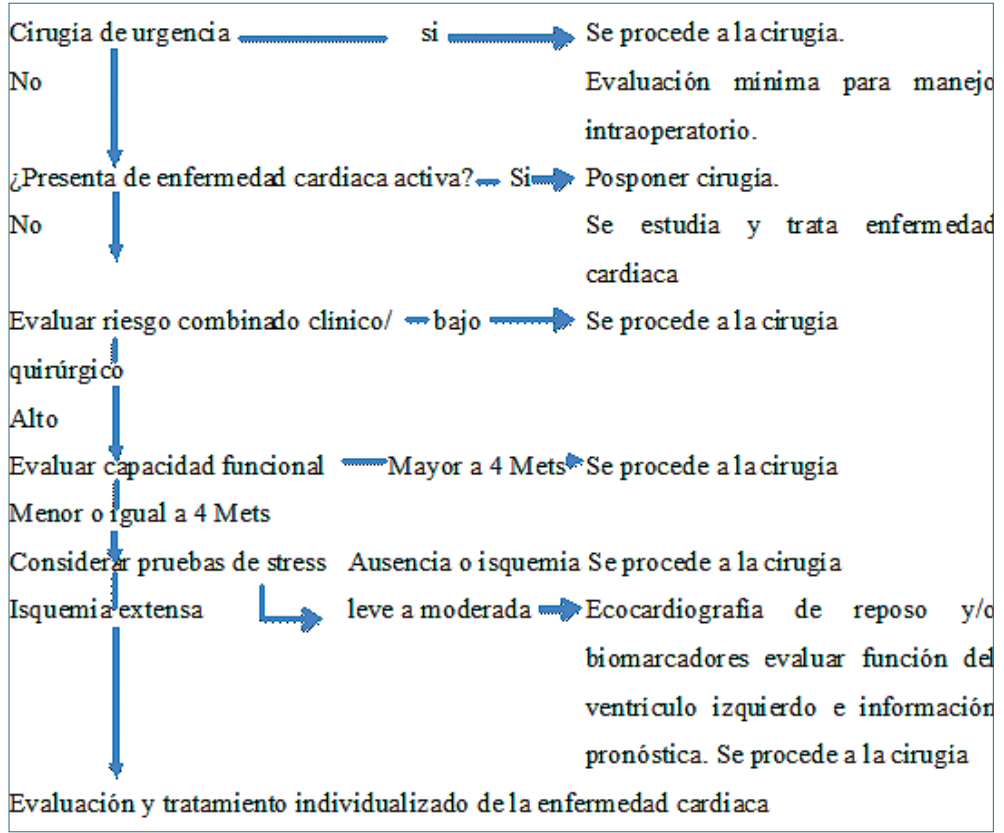

Figura 3. Algoritmo de la AHAIACC. combinación de factores clínicos y el tipo de procedimiento quirúrgico. Se utiliza cualquiera de los 2 índices (NSQIP que tiene incorporado el procedimiento quirúrgico/RCR/ con la clasificación de riesgo quirúrgico)[1]-[3].

\section{Paso 4}

Procedimientos de riesgo intermedio ( $1 \%$ a $5 \%$ ) o alto (mayores de $5 \%$ ). Para evaluar la eventualidad de sufrir un evento cardiaco, es necesario evaluar la capacidad funcional. Si es mayor a 4 METs no será necesario profundizar los estudios y se procede con la cirugía.

En el paso 3 y 4 hay que tener las siguientes consideraciones. En pacientes con uno o más factores clínicos de riesgo, se puede considerar un electrocardiograma basal preoperatorio. Si tiene cardiopatía isquémica conocida puede considerarse el uso de betabloqueadores (BB) y en pacientes con disfunción sistólica el uso de inhibidores de enzima convertidora (IECA). En cirugía vascular debe iniciarse el uso de estatinas (E) precozmente (IIA, nivel de evidencia B).

\section{Paso 5 y 6}

En pacientes con capacidad funcional menor a 4 METs, debe considerarse nuevamente el tipo de procedimiento a realizar. Si es de riesgo intermedio se procede con la cirugía (ESC). Si el procedimiento es de alto riesgo, deben considerarse los factores de riesgo (índice de Lee/RCRI). Con dos o menos se puede con- siderar ecocardiografía en reposo y determinación de biomarcadores para evaluar la función de ventrículo izquierdo y sobre todo obtener información pronóstica con respecto a la posibilidad de eventos cardiacos. Los pacientes que presentan tres o más factores de riesgo deberán realizarse estudio no invasivo de stress cardiaco. Para la ACC/AHA, en cambio, si el paciente tiene elevado riesgo, y la capacidad funcional es mayor a 4 METs se procede con la cirugía. Si es menor a 4 METs o desconocida, debe evaluarse si el profundizar los estudios pudiese cambiar la decisión de cirugía y/o los cuidados perioperatorios. Si la decisión es que sí, lo indicado son los estudios de stress farmacológico.

\section{Paso 7}

$\mathrm{Si}$ el test es negativo o muestra una isquemia leve a moderada, se procede con la cirugía con medidas de protección miocárdica y cuidado perioperatorio específico. Si el resultado es una isquemia extensa, deben continuarse los estudios en vistas a resolver esta patología, considerando el balance riesgo-beneficio de acuerdo al tipo de cirugía planeada. Para la ACC/ $A H A$ en cambio, si el test es negativo se procede con la cirugía y si es positivo, se planteará el tratamiento de la enfermedad coronaria.

\section{Principales diferencias entre las guías}

1- Riesgo de acuerdo al tipo de procedimiento. 
La ESC utiliza la clasificación anterior con posibilidad de sufrir un evento cardiaco de bajo riesgo, menor a $1 \%$, riesgo intermedio entre $1 \%$ y $5 \%$, riesgo alto mayor a 5\%. La AHA las clasifica en bajo riesgo, menor a $1 \%$ y alto riesgo mayor $1 \%[1]-[3]$.

2- En el algoritmo.

La ESC mantiene la evaluación del riesgo quirúrgico como un paso separado. Por lo tanto, la cirugía de bajo riesgo, por ella misma, no necesita profundizar los estudios, en cambio, la AHA evalúa en forma conjunta el riesgo quirúrgico junto a los factores de riesgo clínicos. Para ello utiliza en forma conjunta el $R C R /$ con la clasificación quirúrgica o NSQIP, que ya tiene incorporado el procedimiento quirúrgico.

\section{Exámenes preoperatorios que forman parte de la evaluación cardiaca para cirugía no cardiaca y que aún son motivo de debate}

\section{1- Electrocardiograma (ECG)}

No está recomendado de rutina a todos los pacientes (clase III, evidencia B)[2].

La ESC recomienda la realización de ECG si existe riesgo del paciente y/o de la cirugía, siendo la edad (65 años) un elemento determinante en la decisión. La AHA no lo recomienda en pacientes con o sin factores de riesgo sometidos a cirugía de bajo riesgo. Puede considerarse en pacientes asintomáticos que se someten a cirugía de riesgo intermedio. Finalmente, la $A S A$ al igual que la ESC y $A H A$, proponen que son los factores de riesgo, la presencia de patología y/o el riesgo quirúrgico cardiovascular los determinantes para su solicitud. Rara vez los hallazgos patológicos perioperatorios en pacientes de bajo riesgo llevan a cambios en la conducta perioperatoria.

El mayor estudio prospectivo para abordar el valor pronóstico de un ECG postoperatorio, mostró que los nuevos hallazgos isquémicos fueron un predictor independiente de sucesos cardíacos mayores posteriores (la concordancia entre la isquemia en un ECG y la elevación del nivel de troponina T fue de 85\%[2]-[3].

La guía de la CSC recomienda realizar un ECG postoperatorio en la unidad de cuidados postanestésicos en pacientes con una medición elevada de pro-hormona de péptido natriurético cerebral (NTproBNP)/péptido natriurético cerebral (BNP) antes de la cirugía o, si no hay medición de NT-proBNP/BNP antes de la cirugía, en aquellos que tienen una puntuación de $R C R I \geq 1$, de 45-64 años con enfermedad cardiovascular significativa* o de 65 años o más[4].

*Enfermedades cardiovasculares significativas incluyen antecedentes conocidos de cardiopatía coronaria, enfermedad vascular cerebral, enfermedad vascular periférica, insuficiencia cardíaca congestiva, hipertensión pulmonar severa, estenosis aórtica severa, estenosis mitral severa o miocardiopatía hipertrófica obstructiva severa)[4].

\section{2- Radiografía de tórax}

No está indicada como parte habitual de la evaluación cardiovascular preoperatoria para cirugía no cardiaca. Debe ser solicitada para valorar situaciones específicas: enfermedad cardiaca y/o respiratoria descompensada e infección respiratoria baja[1]-[3].

\section{3- Biomarcadores}

Los más estudiados son las troponinas cardiacas, el péptido natriurético cerebral (BNP) y su prohormona (NT proBNP). Las troponinas T e I (cTnT y cTnl) son indicadores de daño miocárdico y tanto el BNP como el NT proBNP son marcadores de disfunción del ventrículo izquierdo. Se producen en los miocitos cardiacos en respuesta a aumentos del estrés de la pared miocárdica. Esto sucede en cualquier fase de la insuficiencia cardiaca, independiente de la presencia o ausencia de isquemia miocárdica. Las guías europeas y norteamericanas no recomiendan su determinación sistemática, ya que la evidencia no es suficiente. Sin embargo, las guías europeas, recientemente han recomendado su medición en un subgrupo de pacientes de alto riesgo $(\leq 4$ METs o índice revisado de riesgo cardiaco $>1$ para cirugía vascular y $>2$ para cirugía no vascular) antes de la cirugía mayor y 48-72 h después. Las guías de la CSC recomiendan medir NT-proBNP o BNP antes de la cirugía no cardiaca para mejorar la estimación del riesgo cardíaco perioperatorio en pacientes de 65 años de edad o entre 45-64 años de edad con enfermedad cardiovascular significativa o con una puntuación de $R C R I$ $\geq 1$. En comparación con las imágenes cardiacas y las pruebas de esfuerzo cardíaco no invasivo, los biomarcadores de NT-proBNP/BNP son económicos y evitan la necesidad de nuevas imágenes cardiacas y pruebas de stress no invasivas[4]. La elevación de troponinas se asocian a mal pronóstico en muchas patologías cardiacas, pulmonares, sepsis y hemorragia subaracnoidea, por lo que, no permite predecir qué tipo de complicación presentará el paciente. Pueden predecir eventos cardiovasculares en los primeros 30 días de la cirugía vascular, mejoran la utilidad de los índices revisados de riesgo cardiaco y tienen valor predictivo de insuficiencia cardiaca en el postoperatorio, por lo que su determinación podría ser útil en pacientes con alto riesgo de falla cardiaca. Sin embargo, se necesitan más estudios para incorporarlos definitivamente. Varios metaanálisis han mostrado que un nivel ele- 
vado de troponina es un predictor independiente de mortalidad por cualquier causa al año después de la cirugía[3]-[4]. Debido a que la mayoría de los pacientes que sufren un IAM o injuria miocárdica después de una cirugía no cardiaca (MINS) son asintomáticos, la monitorización rutinaria de troponina puede detectar pacientes que tienen un riesgo notablemente mayor de muerte dentro de los 30 días de la cirugía, ya que la mayoría de estos pacientes (84\%) permanecieron asintomáticos y solo se detectaron mediante la vigilancia de rutina de los niveles de troponina. Se publicó recientemente un "Algoritmo Suizo" en nombre de la Sociedad Suiza de Anestesia y Reanimación (SGARSSAR)[5]. Si el riesgo de cirugía, la capacidad funcional y la presencia de factores de riesgo clínico indican un riesgo mayor, BNP se utiliza para diferenciar aún más el riesgo del paciente. Pacientes con niveles normales puede someterse a cirugía sin más pruebas, mientras que los pacientes con elevación de BNP o NT-proBNP son de alto riesgo, deben someterse a un tratamiento cardiológico y la vigilancia de troponinas es recomendado diariamente durante 48 a 72 h. Un cambio de $\geq$ $20 \%$ se interpreta como significativo y se debe diferenciar si la injuria miocárdica es por causa isquémica o no isquémica. Si la causa es isquémica, se procede a la clasificación de infarto tipo I (ruptura de placa) o tipo II (desbalance entre oferta y demanda)[6]. Las guías de la CSC recomiendan obtener diariamente medidas de troponina durante 48-72 h después de la cirugía no cardíaca en pacientes con un riesgo inicial $>5 \%$ de muerte cardiovascular o IAM no mortal 30 días después de la cirugía, es decir, pacientes con una medición elevada de NT-proBNP/BNP antes de la cirugía o, si no hay medición de NT-proBNP / BNP antes de la cirugía, en aquellos que tienen una puntuación $R C R I \geq 1$, de 45-64 años con enfermedad cardiovascular significativa o de 65 años o más[4]. Actualmente, la nueva generación de troponina T ultrasensible (hs $\mathrm{TnT}$ ) en pacientes asintomáticos con riesgo cardiovascular elevado, puede detectar y cuantificar la injuria miocárdica después de cirugía no cardiaca, puede predecir eventos cardiovasculares y estar asociada a mortalidad significativa a 30 días[7]. Nuevos prometedores biomarcadores, tales como copeptina, ácido microribonucleico (MiRNAs), ambos detectados en respuesta al stress endógeno, prometen ser marcadores de inflamación periopertoria, stress e injuria miocárdica en cirugía no cardiaca.

Sin embargo, a pesar de la eficacia demostrada de estos biomarcadores, no es posible realizarlos de forma rutinaria a todos los pacientes, actualmente, no hay un biomarcador que sea $100 \%$ específico y $100 \%$ sensible, y se pueden elevar en condiciones de ausencia real de isquemia miocárdica, por lo que tratar a pacientes por una condición no existente podría ser dañino[6],[7].

Por lo tanto, su uso es recomendable en los casos previamente mencionados, y más que un solo biomarcador, un panel de ellos seguidos por mediciones secuenciales, para predecir eventos cardiacos encubiertos que nos permitan realizar intervenciones perioperatorias (monitorización, elección de drogas, entre otras) adecuadas para predecir y reducir la injuria miocárdica, sobre todo aquellos pacientes de alto riesgo, sometidos a cirugía vascular o de alto riesgo.

\section{4- Ecocardiograma}

Las guías europeas y norteamericanas recomiendan no realizar ecocardiograma preoperatorio de rutina a los pacientes que se someterán a cirugía no cardiaca de bajo a intermedio riesgo, ya que no determinará cambio de conducta[1],[2]. Se recomienda la evaluación de la función del ventrículo izquierdo con ecocardiografía trans-torácica (ETT) en pacientes con sospecha o diagnóstico de falla cardiaca y que están siendo evaluados para cirugía de riesgo intermedio a alto. Una fracción de eyección del ventrículo izquierdo $(\mathrm{FEVI})<30 \%$ es un predictor independiente de resultados adversos perioperatorios y a largo plazo de muerte, en pacientes que se someten a cirugía no cardiaca de alto riesgo[1]-[3].

Sin embargo, la recomendación de un ecocardiograma preoperatorio para evaluar la función del ventrículo izquierdo, considera solo la función sistólica, no tomando en cuenta la evaluación de la función diastólica. Algunos estudios[3],[8] encuentran la disfunción diastólica (DD) del ventrículo izquierdo como factor de riesgo independiente de eventos mayores cardiovasculares en el postoperatorio. Un metaanálisis publicado el 2016 de 3.876 pacientes sometidos a cirugía no cardiaca, reportó que existe una mayor incidencia de eventos adversos cuando el paciente presenta DD en procedimientos que correspondían a cirugía vascular abierta[9]. Si bien la DD aún no es considerada predictor de eventos adversos, es importante tenerla en cuenta a la hora de planificar el manejo perioperatorio, ya que prácticamente la mitad de los pacientes con insuficiencia cardiaca presentan función sistólica preservada y DD exclusiva. Por último, la CSC no recomienda la realización de ecocardiograma de reposo preoperatorio para mejorar la estimación de riesgo cardiovascular perioperatorio, ya que los parámetros ecocardiográficos no resultan en un aumento adicional del riesgo relativo al compararlo con $R C R /$ y el NT pro BNP[4]. 


\section{5- Estudios funcionales de isquemia}

Los estudios funcionales tienen un alto valor predictivo negativo y una prueba negativa se asocia con una incidencia muy baja de eventos cardiacos en pacientes sometidos a cirugía; sin embargo, el valor predictivo positivo es relativamente bajo; esto significa que la probabilidad postquirúrgica de un evento cardiaco es baja. Por lo tanto, la prueba sólo debe realizarse si sus resultados influyen en el pronóstico real perioperatorio. Se recomienda en casos de cirugía de alto riesgo, en pacientes con capacidad funcional $\leq 4$ Mets y más de dos factores clínicos de riesgo[2].

Su indicación debe pasar por tres pasos:

- Valoración de la capacidad funcional.

- Riesgo de la cirugía.

- Score de riesgo (Lee).

En pacientes con mala CF y cirugía de alto riesgo (Figura 4).

\section{6- Estrategias farmacológicas de reducción de riesgo cardiovascular}

La indicación de la administración de BB, ha presentado modificaciones a lo largo de los años. Primero se mostraron beneficios de su implementación desde el preoperatorio[10] y, posteriormente, se evidenciaron efectos deletéreos como hipotensión arterial y accidente cerebrovascular (AVE) (POISE)[11]. Estudios de la serie DECREASE[12] dentro de los cuales se incluyen varios trabajos de Poldermans han suscitado reparos en la comunidad médica por discrepancias metodológicas muy importantes. Las guías también excluyen el estudio POISE. La argumentación, compleja por supuesto, es que se utilizó una dosis excesiva de BB (metoprolol). La revisión Cochrane año 2014 muestra que la utilización de BB en pacientes de bajo riesgo determinaría un aumento de la mortalidad de todo tipo, mientras que, en pacientes de alto riesgo, el nivel de evidencia es bajo a moderado (clase II, evidencia B)[1],[2]. En cirugía no cardiaca la utilización de $B B$, iniciados dentro de las $24 \mathrm{~h}$ previas a la cirugía no cardiaca, se asocia a un posible aumento de la mortalidad y AVE, a una menor incidencia de IAM y arritmias supraventriculares, así como una mayor frecuencia de hipotensión y bradicardia. Actualmente los BB se deben continuar solamente en pacientes que serán sometidos a cirugía y que los reciben crónicamente (I, nivel de evidencia B)[1]-[4]. Se pueden recomendar en pacientes con factores de riesgo alto, en cirugía de alto riesgo o ASA > 3 y en pacientes con cardiopatía isquémica o isquemia miocárdica diagnosticada al inicio del tratamiento preoperatorio (Illb, nivel de evidencia B)[1]-[3]. La CSC no recomienda el inicio de BB dentro de las $24 \mathrm{~h}$ previas a la cirugía no cardíaca[4]. La decisión de cuando comenzar su uso debe ser idealmente semanas previas a la cirugía no cardiaca y debe evaluarse cuidadosamente el riesgo de AVE o de otras contraindicaciones relativas. Es importante destacar, que el $A V E$, aunque tiene alta morbilidad, es menos frecuente que un evento cardiaco adverso mayor[2],[3].

El mecanismo por el cual la terapia con E parece ser beneficiosa antes de la cirugía para reducir los eventos cardiacos, no es del todo claro, pero puede estar relacionado con los efectos pleiotrópicos, así como una acción hipolipemiante[2],[3]. Según las guías actuales, la mayoría de los enfermos con enfermedad arterial periférica deben recibir tratamiento con E. Si son candidatos a cirugía vascular abierta o a una intervención endovascular, el tratamiento debe mantenerse después de la intervención. En pacientes vasculares no tratados con $E$, es deseable iniciar el tratamiento al menos dos semanas antes de la intervención y mantenerlo durante al menos un mes tras ella[1]-[3]. La CSC, basada en una revisión sistemática y metaanálisis de tres estudios acerca del inicio de tra-

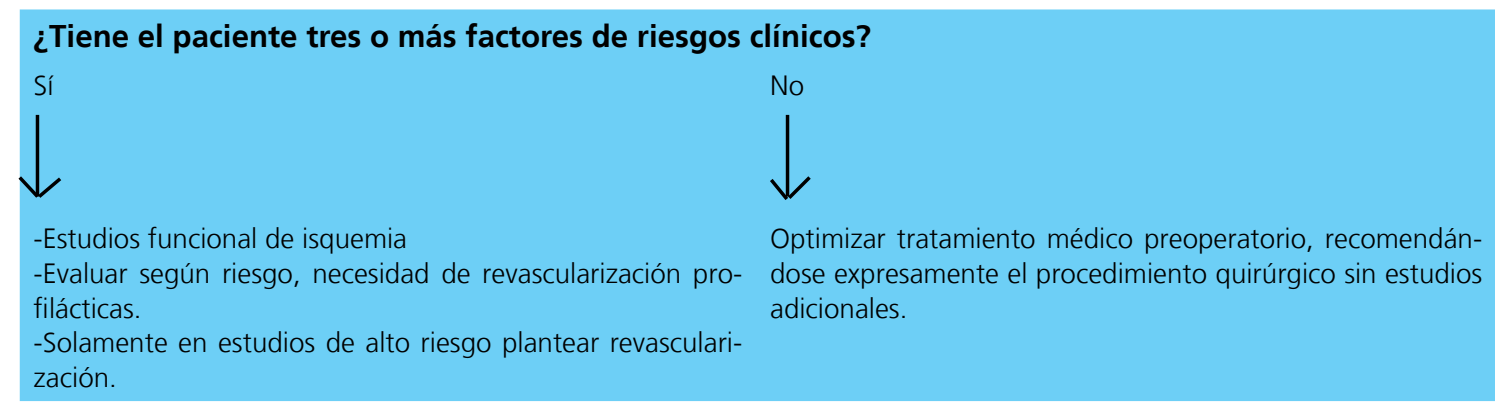

Figura 4. Algoritmo de indicación de estudios. Basada en perioperatorio del paciente con cardiopatía de Riva y González et al. Por último, la CSC no recomienda la realización de estudios funcionales de isquemia. Su costo y potenciales demoras en su realización deben tenerse en cuenta. Hay ausencia de evidencia de una mejora absoluta en la reclasificación del riesgo ${ }^{4}$. 
tamiento con E en pacientes que van a cirugía vascu$\operatorname{lar}[13]$, no recomiendan su uso previo a la cirugía no cardiaca, pero sí su mantención en los pacientes que la toman crónicamente[4].

Con el ácido acetilsalicílico (ASA), debe considerarse el riesgo de complicaciones tromboembólicas y/o cardiovasculares versus el sangrado perioperatorio, en especial en cirugías de columna vertebral, neurológicas y oftalmológicas e individualizarse su administración en pacientes de alto riesgo. La CSC recomienda no iniciar ni continuar ASA para la prevención de eventos cardíacos perioperatorios, excepto en pacientes con un stent coronario reciente, y pacientes sometidos a endarterectomía carotídea[4]. En pacientes que la toman permanentemente recomiendan suspenderla tres días antes de la cirugía y reiniciarla cuando el riesgo de sangrado relacionado con la cirugía haya pasado.

Las guías de la CSC recomiendan el inicio de ASA y $E$ a largo plazo en pacientes que sufren MINS o un IAM después de una cirugía no cardiaca, aunque estos datos se limitan solo a datos observacionales[4].

No se recomienda el inicio de alfa 2 agonistas en la prevención de eventos cardiacos en cirugía no cardiaca, ya que aumenta el riesgo de hipotensión, bradicardia e IAM no fatal[4].

Los estudios sobre el efecto perioperatorio de los bloqueadores de los canales de calcio son escasos. Debido a ello, no se recomienda su uso en la prevención de eventos cardiacos en cirugía no cardiaca[4].

Debe considerarse mantener el tratamiento con los inhibidores de la enzima de angiotensina (IECA) y los antagonistas del receptor de angiotensina II (ARA II) bajo estrecha vigilancia durante la cirugía no cardiaca de pacientes estables con insuficiencia cardiaca y disfunción sistólica del VI (Ila, evidencia C). Es razonable la suspensión temporal del tratamiento con IECA o ARA II antes de la cirugía no cardiaca de pacientes hipertensos (Ila, evidencia C), a pesar de que las guías recomiendan continuar la administración del fármaco $(A C C / A H A)$. Esta recomendación se basa en estudios pequeños y observacionales retrospectivos. Existe el riesgo de hipotensión particularmente tras la inducción de la anestesia y el uso concomitante de
$B B[3]$. Es así, que la CSC recomienda suspender los IECA/ARA II empezando $24 \mathrm{~h}$ antes de la cirugía no cardíaca en pacientes tratados crónicamente. Debido a que el riesgo de hipotensión es mayor dentro de las $24 \mathrm{~h}$ de la cirugía, los médicos deben considerar reiniciar IECAVARA II el día dos después de la cirugía en pacientes que reciben terapia crónica, si el paciente está hemodinámicamente estable[4].

Se estima que el 5\%-25\% de los pacientes con stents coronarios pueden requerir cirugía no cardíaca dentro de los 5 años posteriores a la implantación del stent[14]. En el manejo de pacientes en tratamiento antiagregante doble (DAPT) derivados para cirugía no cardiaca hay que considerar:

1- El riesgo de trombosis del stent, particularmente si el DAPT necesita ser interrumpido vs las consecuencias de retrasar el procedimiento quirúrgico.

2- El aumento del riesgo de hemorragia perioperatoria y posibles consecuencias de sangrado si se continúa con DAPT[14].

Se requiere un enfoque multidisciplinario, cardiólogos, anestesistas, cirujanos y hematólogos, para determinar el riesgo potencialmente mortal de complicaciones hemorrágicas versus trombosis del stent del paciente. Luego decidir la mejor estrategia.

El grupo Stent After Surgery, recientemente, ha propuesto una clasificación práctica del riesgo de hemorragia asociado con cada tipo de cirugía no cardiaca[15]. Esta clasificación (Tablas 3 y 4) está basada en recomendaciones según el procedimiento quirúrgico en lugar de un perfil hemorrágico del paciente.

En procedimientos quirúrgicos con bajo riesgo de hemorragia, se deben tomar todas las medidas posibles para no interrumpir la DAPT en el preoperatorio. En procedimientos quirúrgicos con riesgo de sangrado moderado, los pacientes deben mantenerse con Aspirina, mientras que el tratamiento con inhibidores del receptor plaquetario de adenosina difosfato (P2Y12) debe suspenderse de ser posible. Los pacientes con DAPT que se someten a cirugías no cardíacas de alto riesgo de sangrado (incluyendo reconstrucciones vasculares, procedimientos viscerales complejos, neurocirugía y operaciones transbronquiales)[16], se debe prestar especial atención a la interrupción oportuna

Tabla 3. Riesgo de sangrado en cirugía general del grupo Stent After Surgery

\begin{tabular}{lll}
\hline Riesgo bajo & Riesgo intermedio & Riesgo alto \\
Hernioplastia, cirugía plástica de her- & Hemorroidectomía, esplenectomía, & $\begin{array}{l}\text { Resección hepática, duodenocefalo- } \\
\text { nias incisionales, colecistectomía, apen- }\end{array}$ \\
$\begin{array}{l}\text { dicectomía, colectomía, resección gás- } \\
\text { trica e intestinal, cirugía de mama }\end{array}$ & resección rectal, tiroidectomía & \\
\hline
\end{tabular}




\section{Tabla 4. Riesgo de sangrado en cirugía vascular del grupo Stent After Surgery}

\begin{tabular}{lll}
\hline Riesgo bajo & Riesgo intermedio & Riesgo alto \\
$\begin{array}{l}\text { Endarterectomía carotidea, bypass o } \\
\text { endarterectomía de extremidades infe- }\end{array}$ & Cirugía de la aorta abdominal abierta & $\begin{array}{l}\text { Cirugía abierta torácica y toracoabdo- } \\
\text { minal }\end{array}$ \\
$\begin{array}{l}\text { rirores. *EVAR, **TEVAR, amputación } \\
\text { de miembros }\end{array}$ & \\
\hline
\end{tabular}

*EVAR: reparación endovascular de aneurisma de la aorta.

**TEVAR: reparación endovascular de aneurisma de la aorta torácica.

de la terapia con inhibidores de P2Y12 para minimizar el período de no terapia antes de la intervención quirúrgica.

La duración estándar de la DATP es diferente tras el implante de stent no recubiertos (un mes) que tras el stent liberador de fármacos (entre tres meses a un año dependiendo de la generación). La indicación de colocación del stent, es lo que condiciona la decisión. Es muy diferente si el paciente tiene un síndrome coronario agudo (SCA) vs uno con una enfermedad coronaria crónica estable y debe someterse a cirugía no cardiaca[14].

La cirugía per se, independiente del momento de interrupción del DAPT, se asocia con efectos proinflamatorios y protrombóticos, lo que aumenta el riesgo de trombosis coronaria a nivel del segmento vascular del stent, así como a lo largo de la vasculatura coronaria[15]. Por lo tanto, en pacientes sometidos a cirugía no cardíaca después de SCA reciente o implantación de stent, los beneficios de la cirugía temprana para una patología específica (ej; tumores malignos o reparación de aneurismas vasculares), deben equilibrarse con el riesgo de eventos cardiovasculares y la estrategia debe ser discutida por un equipo multidisciplinario.

En el SCA, pacientes que presentaron un IAM u otras características de alto riesgo de isquemia (Tabla 5) y que requieren DAPT, la cirugía electiva puede posponerse hasta 6 meses (evidencia llb), independiente del tipo de stent colocado, no así en aquellos con enfermedad coronaria crónica estable, en que después de la implantación del stent coronario, puede considerarse cirugía electiva que requiere la interrupción del inhibidor de P2Y12 después de 1 mes, independientemente del tipo de stent. La Aspirina puede mantenerse durante todo el período perioperatorio (evidencia lla)[15].

Ante la eventualidad de ser necesaria una cirugía no cardiaca antes del plazo ideal y en el que el riesgo quirúrgico de hemorragia es excesivamente alto con DAPT, ambos agentes antiplaquetarios orales deben suspenderse en el perioperatorio. Se puede considerar una estrategia puente con los agentes antiplaquetarios intravenosos (evidencia llb)[15],[17],[18],[19],[20]. Para pacientes de alto riesgo de trombosis de stent, se puede realizar terapia puente con inhibidores de la glicoproteína Iribú/llla al suspender el inhibidor P2Y12 (clopidroguel, ticagrelor y prasugrel) y la cirugía se debe realizar en un hospital que cuente con angioplastia de urgencia. Cabe destacar que el Cangrelor, un inhibidor de P2Y12 intravenoso reversible, proporciona una inhibición plaquetaria eficaz y es una alternativa atractiva a los inhibidores de la glucoproteína Ilb/llla[15],[19].

La interrupción de los inhibidores de P2Y12 se debe considerar al menos 3 días antes de la cirugía para ticagrelor, al menos 5 días para clopidogrel, y al menos 7 días para prasugrel (IIa)[8].

Si la terapia con inhibidores $\mathrm{P} 2 \mathrm{Y} 12$ se detuvo antes de un procedimiento quirúrgico, la terapia debe reiniciarse tan pronto como sea posible (dentro de $48 \mathrm{~h}$ ) dado el riesgo trombótico significativo asociado con la falta de inhibición plaquetaria temprana[15].

\section{Consideraciones anestésicas y manejo intraoperatorio}

Persiste la controversia acerca del impacto que puede tener la elección de la anestesia sobre la incidencia y gravedad de las complicaciones cardiovasculares.

Los procedimientos anestésicos, consideran: anestesia local, regional (neuroaxial o bloqueos de nervios periféricos), sedación intravenosa monitorizada y anestesia general.

Según las guías europeas, la técnica anestésica influye poco en los resultados cardiovasculares postoperatorios, siempre y cuando se mantengan las variables hemodinámicas dentro de ciertos parámetros preestablecidos. La AHA refiere que no hay evidencia que sugiera beneficio cardioprotector en el uso o adición de anestesia neuroaxial para el manejo anestésico intraoperatorio en cirugía no cardiaca. Todos los agentes anestésicos volátiles o intravenosos, son 
razonables para los pacientes sometidos a cirugía no cardiaca y la elección estará determinada por factores distintos a la prevención de la cardiopatía isquémica en el perioperatorio (Ila: A). El pre-condicionamiento isquémico con anestésicos halogenados, ha sido demostrado en cirugía cardiaca, sin embargo, este efecto benéfico no ha sido demostrado para otro tipo de cirugía[1].

El sistema cardiovascular sufre varias injurias durante el perioperatorio: estrés prequirúrgico, maniobras invasivas como laringoscopia e intubación, magnitud de la injuria quirúrgica, modificaciones de la volemia, duración de la cirugía, hipotermia, drogas anestésicas, entre otras.

La mayoría de las técnicas anestésicas reducen el tono simpático, lo que produce diminución del retorno venoso por aumento de la distensibilidad del sistema venoso, vasodilatación arterial y como consecuencia reducción de la presión arterial[21]. El manejo anestésico debe garantizar el mantenimiento del flujo sanguíneo y la presión de perfusión a los órganos. La evidencia más reciente indica que no existe un valor objetivo universal de presión arterial en el intraoperatorio, pero los descensos de la presión arterial media (PAM) basal > 20\% o menores a $60 \mathrm{mmHg}$ mantenidos durante $30 \mathrm{~min}$ (Ilb, evidencia B) o más, se asocian a un aumento estadísticamente significativo del riesgo de complicaciones postoperatorias, incluyendo IAM, accidente cerebrovascular (ACV) y muerte[2],[3].

Los efectos hemodinámicos sistémicos de los anestésicos volátiles son complejos ya que están determinados por la interacción de sus efectos en el miocardio, acciones directas en la vasculatura arterio-venosa y alteraciones en la actividad del sistema nervioso autónomo. Los anestésicos volátiles producen depresión del inotropismo de los ventrículos, disminución de la función diastólica ventricular izquierda y del acoplamiento del ventrículo izquierdo con el sistema arterial. La severidad de estos efectos es dosisdependiente y están relacionados con alteraciones de la homeostasis del calcio intracelular en el miocito cardiaco[21]. También, se afectan los determinantes de la postcarga del ventrículo izquierdo en diversos grados tanto en el miocardio normal como disfuncional, sensibilizan el miocardio a los efectos arritmogénicos de la adrenalina en diversos grados y pueden facilitar el desarrollo de arritmias auriculares o ventriculares durante la isquemia o IAM. También son agentes vasodilatadores coronarios relativamente débiles.

La anestesia neuroaxial, se caracteriza por un bloqueo intenso del sistema simpático. El bloqueo de las fibras nerviosas produce pérdida del tono vasomotor, lo que determina cambios considerables en el flujo sanguíneo dependiendo del nivel del bloqueo alcanzado. La anestesia neuroaxial que alcanza las raíces torácicas simpáticas $1^{\circ}$ a $5^{\text {ta }}$, determinan bloqueo de las fibras cardiacas simpáticas aferentes con pérdida de cronotropismo e inotropismo, mientras que la restringida al nivel torácico bajo y lumbar dan como resultado el bloqueo simpático periférico con dilatación vascular en la pelvis y las extremidades inferiores[3].

Según las guías europeas, la técnica anestésica influye poco en los resultados cardiovasculares postoperatorios siempre y cuando se mantengan las variables hemodinámicas dentro de ciertos parámetros preestablecidos. La AHA refiere que no hay evidencia que sugiera beneficio cardioprotector en el uso o adición de anestesia neuroaxial para el manejo anestésico intraoperatorio en pacientes elegibles para anestesia neuroaxial intraoperatoria en cirugía no cardiaca. De la misma manera, no hay evidencia favorable con el uso de cualquiera de los agentes anestésicos volátiles o intravenosos. Todos pueden usarse, y la elección estará determinada por factores distintos a la prevención de la cardiopatía isquémica en el (Ila: A). A pesar de que el uso de agentes anestésicos volátiles ha demostrado en cirugía cardiaca, una reducción de isquemia miocárdica o de IAM, ello no ha sido demostrada en cirugía no cardiaca[1].

Cuando se compara la anestesia regional vs general, se concluye que no hay un beneficio de una sobre la otra, no obstante, se recomienda con un modesto nivel de evidencia (II; B)[1],[3] y teniendo en cuenta el riesgo vs beneficio. En el mismo sentido y con evidencia (Ila; B), la AHA recomienda la analgesia neuroaxial para alivio del dolor postoperatorio en pacientes sometidos a cirugía de aorta abdominal con el objetivo de disminuir la incidencia de IAM perioperatorio.

Una revisión Cochrane[22] y otros estudios recientes establecen que para pacientes de riesgo cardiovascular moderado y alto, las técnicas regionales reducen la mortalidad, con un nivel de evidencia moderado. Sin embargo, esta reducción no es debida a disminución en las complicaciones cardiovasculares, sino que, probablemente, a otras respiratorias (neumonía) y/o tromboembólicas, principalmente.

La Sociedad Americana del Dolor en las guías de 2016, recomienda considerar de forma rutinaria el uso de analgesia epidural o espinal para el tratamiento del dolor postoperatorio en pacientes que se someten a procedimientos torácicos y abdominales mayores: cesárea, cirugía de cadera y miembros inferiores, en pacientes con riesgo de complicaciones cardiacas, pulmonares o íleo prolongado[9]. 


\section{Algunas consideraciones acerca de la monitorización con ecocardiografía intraoperatoria (TEE) en cirugía no cardiaca}

Si bien el TEE permite evaluar la función biventricular y valvular, las estructuras intracardiacas, el espacio pericárdico, la aorta torácica, su uso está ampliamente demostrado en cirugía cardiaca, su evidencia de uso en cirugía no cardiaca es limitada al evaluar la función miocárdica regional y su asociación con cualquier evento cardiaco adverso. El uso rutinario en cirugía no cardiaca para evaluar anormalidades cardiacas o monitorizar isquemia miocárdica no está recomendado en pacientes sin factores de riesgo o procedimientos quirúrgicos con riesgo de significativo compromiso hemodinámico, pulmonar o neurológico[1]. En contraste, su uso de emergencia es razonable en pacientes para determinar la causa inexplicable y severa de inestabilidad hemodinámica, a pesar de terapia correctiva[23], siempre y cuando sea manejado por un experto[24]. En nuestro país, no hay suficiente gente entrenada y acreditada para realizar estos procedimientos.

\section{Puntos clave}

1.- La valoración clínica sigue siendo uno de los pilares fundamentales en la evaluación del riesgo anestésico-quirúrgico.

2.- Existen scores de riesgo en la valoración preoperatoria incorporados en las guías del American College of Cardiology, la American Heart Association y la European Society of Cardiology.
3.- La Canadian Cardiovascular Society entrega recomendaciones, muchas de ellas diferentes de las pautas descritas previamente.

4.- Sin embargo, el Revised Cardiac Risk Index o índice de Lee, es el que mayoritariamente se aplica en la práctica clínica. Es el más antiguo, desarrollado y validado a través de muchos años. Presenta seis factores clínicos de riesgo independiente, por sobre los otros puntajes de predicción de riesgo clínico disponibles.

5.- El nivel de evidencia en relación a la cardio-protección farmacológica es moderado.

6.- En el manejo de pacientes coronarios en tratamiento antiagregante doble, derivados para cirugía no cardiaca, hay que considerar: el riesgo de trombosis del stent, las consecuencias de retrasar el procedimiento quirúrgico y el aumento del riesgo de hemorragia.

7.- Hasta la fecha no existe evidencia acerca de cuál es el mejor manejo anestésico que disminuya las complicaciones cardiovasculares perioperatorias en este grupo de pacientes.

\section{Conclusiones}

Todos los modelos de riesgo son importantes, sin embargo, son solo eso, estudios en grandes poblaciones que entregan un valor para calcular morbilidad y mortalidad en diferentes poblaciones. Los modelos de riesgo no deben dictar las decisiones sobre el manejo de un paciente, sino que debe considerárseles un elemento más en la evaluación clínica del perioperatorio.

\section{Referencias}

1. Fleisher LA, Fleischmann KE. Auerbach AD y cols. American College of Cardiology, American Heart Association. 2014 ACC/ AHA guideline on perioperative cardiovascular evaluation and management of patients undergoing noncardiac surgery: a report of the American College of. Cardiology/American Heart Association Task Force on practice guidelines. J Am Coll Cardiol. 2014;64:e77-137. https://doi. org/10.1016/j.jacc.2014.07.944
PMID:25091544

2. Kristensen ST, Knuuti J, Saraste A, Anker S, Bøtker HE, Hert SD, et al. Grupo de Trabajo Conjunto sobre cirugía no cardiaca: Evaluación y manejo cardiovascular de la Sociedad Europea de Cardiología (ESC) y la European Society of Anesthesiology (ESA). Rev Esp Cardiol. 2014;67(12):1052. https://doi.org/10.1016/j.recesp.2014.11.001.

3. Riva J, González L. Texto Perioperatorio del paciente con cardiopatía. Editorial BiblioMédica; 2017.
4. Duceppe E, Parlow J, MacDonald $P$, Lyons K, McMullen M, Srinathan $\mathrm{S}$, et al. Canadian Cardiovascular Society Guidelines on Perioperative Cardiac Risk Assessment and Management for Patients Who Undergo Noncardiac Surgery. Can J Cardiol. 2017 Jan;33(1):17-32. https://doi. org/10.1016/j.cjca.2016.09.008 PMID:27865641

5. Filipovic $\mathrm{M}$, Kindler $\mathrm{CH}$, Walder B. Anästhesiologie und Reanimation: Perioperative kardiale Abklärung und Therapie im Vorfeld nicht-herzchirurgischer 
Eingriffe. Swiss Med. Forum Schweiz. Med. 2018, 18,10781080.

6. Yurttas T, Hidvegi R, Filipovic M. Biomarker-Based Preoperative Risk Stratification for Patients Undergoing NonCardiac Surgery. J Clin Med. 2020 Jan;9(2):351. https:// doi.org/10.3390/jcm9020351 PMID:32012699

7. Dhir S, Dhir A. Cardiovascular Risk Assessment for Noncardiac Surgery: Are We Ready for Biomarkers? J Cardiothorac Vasc Anesth. 2019;00:1-11. PMID:31866221

8. Matyal $R$, Hess PE, Subramaniam B, Mitchell J, Panzica PJ, Pomposelli $F$, et al. Perioperative diastolic dysfunction during vascular surgery and its association with postoperative outcome. Vasc Surg. 2009;50(1):706. https://doi.org/10.1016/j. jvs.2008.12.032.

9. Fayad A, Ansari MT, Yang H, Ruddy T, Wells GA. Perioperative Diastolic Dysfunction in Patients Undergoing Noncardiac Surgery Is an Independent Risk Factor for Cardiovascular Events: A Systematic Review and Meta-analysis. Anesthesiology. 2016 Jul;125(1):7291. https://doi.org/10.1097/ ALN.0000000000001132 PMID:27077638

10. Poldermars D, Boersma E, Bax JJ, $y$ cols. The effect of bisoprolol on perioperative mortality and myocardial infarction in high risk patientes undergoing vascular surgery. N J Med. 1999;341:178994. https://doi.org/10.1056/ NEJM199912093412402.

11. Devereaux PJ, Yang H, Yusuf S, Guyatt G, Leslie K, Villar JC, et al.; POISE Study Group. Effects of extended-release metoprolol succinate in patients undergoing non-cardiac surgery (POISE trial): a rando- mised controlled trial. Lancet. 2008 May;371(9627):1839-

47. https://doi.org/10.1016/ S0140-6736(08)60601-7 PMID:18479744

12. Don Poldermans, Olaf Schouten, Jeroen Bax y cols. Reducing cardiac risk in non-cardiac surgery: evidence from the DECREASE studies, European Heart Journal Supplements, Volume 11, Issue suppl_A, 1 March 2009, Pages A9-A14, https://doi.org/10.1093/ eurheartj/sup004.

13. Sanders RD, Nicholson A, Lewis $S R$, Smith AF, Alderson P. Perioperative statin therapy for improving outcomes during and after noncardiac vascular surgery. Cochrane Database Syst Rev. 2013 Jul;7(7):CD009971. https:// doi.org/10.1002/14651858. CD009971.pub2 PMID:23824754

14. Valgimigli $M$, Bueno $H$, Byrne $R$, y cols. The Task Force for dual antiplatelet therapy in coronary artery disease of the European Society of Cardiology (ESC) and of the European Association for Cardio-Thoracic Surgery (EACTS). Eur Heart J. 2017;0:1-48.

15. Rossini R, Musumeci G, Visconti LO, Bramucci E, Castiglioni B, De Servi S, et al.; Italian Society of Invasive Cardiology (SICI-GISE); Italian Association of Hospital Cardiologists (ANMCO); Italian Society for Cardiac Surgery (SICCH); Italian Society of Vascular and Endovascular Surgery (SICVE); Italian Association of Hospital Surgeons (ACOI); Italian Society of Surgery (SIC); Italian Society of Anaesthesia and Intensive Care Medicine (SIAARTI); Lombard Society of Surgery (SLC); Italian Society of MaxiIlofacial Surgery (SICMF); Italian Society of Reconstructive Plastic Surgery and Aesthetics (SICPRE); Italian Society of Thoracic Surgeons (SICT); Italian Society of Urology (SIU); Italian Society of Orthopaedics and Traumatology (SIOT); Italian Society of Periodontology (SIdP); Italian Federation of Scientific Societies of Digestive System Diseases Lombardia (FISMAD); Association of Obstetricians Gynaecologists Italian Hospital Lombardia (AOGOI); Society of Ophthalmology Lombardia (SOL). Perioperative management of antiplatelet therapy in patients with coronary stents undergoing cardiac and non-cardiac surgery: a consensus document from Italian cardiological, surgical and anaesthesiological societies. Eurolntervention. 2014 May; 10(1):38-46. https:// doi.org/10.4244/EIJV10I1A8 PMID:24832636

16. Moore M, Power M. Perioperative hemorrhage and combined clopidogrel and aspirin therapy. Anesthesiology. 2004 Sep;101(3):792-4. https:// doi.org/10.1097/00000542200409000-00030 PMID:15329606

17. Savonitto S, D'Urbano M, Caracciolo M, Barlocco F, Mariani G, Nichelatti M, et al. Urgent surgery in patients with a recently implanted coronary drugeluting stent: a phase II study of 'bridging' antiplatelet therapy with tirofiban during temporary withdrawal of clopidogrel. $\mathrm{Br}$ J Anaesth. 2010 Mar;104(3):28591. https://doi.org/10.1093/bja/ aep373 PMID:20047898

18. De Servi S, Morici N, Boschetti $E$, Rossini R, Martina P, Musumeci $G$, et al. Bridge therapy or standard treatment for urgent surgery after coronary stent implantation: analysis of 314 patients. Vascul Pharmacol. 2016 May;80:85-90. https://doi. org/10.1016/j.vph.2015.11.085 PMID:26657879

19. Burger W, Chemnitius JM, Kneissl GD, Rücker G. Low-dose aspirin 
for secondary cardiovascular prevention - cardiovascular risks after its perioperative withdrawal versus bleeding risks with its continuation - review and meta-analysis. J Intern Med. 2005 May;257(5):399414. https://doi.org/10.1111/ j.1365-2796.2005.01477.x PMID:15836656

20. Reves JG. Peter s, Glass A, Miller RD. Volatile Anesthetics. Ronald Miller. 7th ed. Churchill Livingstone-Elsseiver; 2009. pp. 719-68.

21. Guay J, Choi P, Suresh S, Albert N, Kopp S, Pace NL. Neuraxial blockade for the prevention of postoperative mortality and major morbidity: an overview of Cochrane systematic reviews. Cochrane Database Syst Rev. 2014 Jan;(1):CD010108. https:// doi.org/10.1002/14651858. CD010108.pub2
PMID:24464831

22. Chou R, Gordon DB, de LeonCasasola OA, Rosenberg JM, Bickler S, Brennan T, et al. Management of Postoperative Pain: A Clinical Practice Guideline From the American Pain Society, the American Society of Regional Anesthesia and Pain Medicine, and the American Society of Anesthesiologists' Committee on Regional Anesthesia, Executive Committee, and Administrative Council. J Pain. 2016 Feb;17(2):131-57. https://doi. org/10.1016/j.jpain.2015.12.008 PMID:26827847

23. Reeves ST, Finley AC, Skubas NJ, Swaminathan M, Whitley WS, Glas KE, et al. Council on Perioperative Echocardiography of the American Society of Echocardiography; Society of Cardiovascular Anesthesiologists. Basic perioperative transesophageal echocardiography examination: a consensus statement of the American Society of Echocardiography and the Society of Cardiovascular Anesthesiologists. J Am Soc Echocardiogr. 2013 May;26(5):443-56. https://doi. org/10.1016/j.echo.2013.02.015 PMID:23622926

24. American Society of Anesthesiologists and Society of Cardiovascular Anesthesiologists Task Force on Transesophageal Echocardiography. Practice guidelines for perioperative transesophageal echocardiography. An updated report by the American Society of Anesthesiologists and the Society of Cardiovascular Anesthesiologists Task Force on Transesophageal Echocardiography. Anesthesiology. 2010 May;112(5):108496. PMID:20418689 